\title{
Millisecond Functional Dynamics of RNA Polymerases Elucidated by Markov State Models
}

\author{
Xuhui Huang \\ The Hong Kong University of Science and Technology, Kowloon, Hong Kong S.A.R. (China); \\ xuhuihuang@ust.hk
}

Protein conformational dynamics play an important role in numerous biological processes. Markov State Models (MSMs) provide a powerful approach to study these dynamic processes by predicting long time scale dynamics based on many short molecular dynamics (MD) simulations. To improve the efficiency of MSMs, we recently developed quasi-MSM (qMSM) that encodes the non-Markovian dynamics in a generally time-dependent memory kernel. We successfully applied qMSMs to elucidate molecular mechanisms of DNA loading into a bacterial RNA polymerase complex via flexible loading gate (consisting of the clamp and $\beta$-lobe domain), a process occurs at millisecond. Using qMSMs, we showed that the opening of $\beta$-lobe is orders of magnitude faster than that of the clamp, which depends on the structure of the Switch 2 region. Strikingly, opening of the $\beta$-lobe is sufficient geometrically to accommodate DNA loading even when the clamp is partially closed. These two observations highlight $\beta$-lobe's critical role allowing DNA loading during initiation. In my talk, I will also present our recent results in elucidating molecular mechanisms of 1'-Ribose cyano substitution allows Remdesivir to effectively inhibit nucleotide addition of the SARS-CoV-2 RNA-dependent RNA polymerase (RdRp).

Keywords: Molecular Dynamics, RNA polymerase, SARS-CoV-2 RdRp 